\title{
Tunable Plasmonic Silver Nanodomes for Surface-Enhanced Raman Scattering
}

\author{
Mehmet Kahraman ${ }^{1} \cdot$ Ayse Ozbay $^{1} \cdot$ Handan Yuksel $^{2} \cdot$ Ramazan Solmaz $^{2}$. \\ Baran Demir $^{3}$ - Humeyra Caglayan ${ }^{3}$
}

Received: 5 February 2017 / Accepted: 21 March 2017 / Published online: 5 April 2017

(C) Springer Science+Business Media New York 2017

\begin{abstract}
Surface-enhanced Raman scattering (SERS) is an emerging analytical method used in biological and nonbiological structure characterization. Since the nanostructure plasmonic properties is a significant factor for SERS performance, nanostructure fabrication with tunable plasmonic properties are crucial in SERS studies. In this study, a novel method for fabrication of tunable plasmonic silver nanodomes (AgNDs) is presented. The convective-assembly method is preferred for the deposition of latex particles uniformly on a regular glass slide and used as a template for polydimethylsiloxane (PDMS) to prepare nanovoids on a PDMS surface. The obtained nanovoids on the PDMS are used as a mold for AgNDs fabrication. The nanovoids are filled with Ag deposition by the electrochemical method to obtain metallic AgNDs. Scanning electron microscopy (SEM) and atomic force microscopy (AFM) are used for characterization of the structural properties of all fabricated AgNDs. The optical properties of AgNDs are characterized with the evaluation of SERS activity of 4-aminothiphonel and rhodamine 6G. In addition to experimental characterizations, the finite difference time domain (FDTD) method is used for the theoretical plasmonic properties calculation of the AgNDs. The experimental and theoretical results show that the SERS performance of AgNDs is
\end{abstract}

Mehmet Kahraman

mkahraman@gantep.edu.tr; mskahraman46@gmail.com

1 Department of Chemistry, Faculty of Arts and Sciences, Gaziantep University, 27310 Gaziantep, Turkey

2 Department of Chemistry, Faculty of Arts and Sciences, Bingöl University, 12000 Bingöl, Turkey

3 Bilkent University Nanotechnology Research Center, 06800 Ankara, Turkey strongly dependent on the heights and diameters of the AgNDs.

Keywords Tunable $\cdot$ Plasmonic $\cdot$ SERS $\cdot$ Silver

Nanodomes $\cdot$ Electrochemical deposition $\cdot$ FDTD method

\section{Introduction}

An emerging research area called plasmonics focuses on the behavior of collective free electron oscillations at a metaldielectric interface excited by electromagnetic radiation known as surface plasmons (SPs) [1-5]. In recent years, the combination of plasmonics with chemistry has received much attention due to its potential analytical applications such as localized surface plasmon resonance (LSPR) spectroscopy [6], surface plasmon resonance (SPR) spectroscopy [7, 8], and surface-enhanced Raman spectroscopy (SERS) [9].

SERS is a powerful technique that uses the enhancement of the Raman signal of molecules situated in the close vicinity of metallic nanostructures to obtain detailed information regarding the identity of those molecules [10-12]. The literature presented that SERS enhancement is a result of both electromagnetic enhancement $[13,14]$ and chemical enhancement mechanisms $[15,16]$. The excitation of the SPs of noble metal nanostructures plays a major role on electromagnetic enhancement. When a Raman scattering molecule is subjected to intense electromagnetic fields generated on metal surfaces, the higher electric field intensity results in stronger polarization of the molecule and thus the higher induced dipole moment is acquired. This enhancement is proportional to Raman scattered light intensity and considered as the major component of the enhancement mechanism [17]. On the other hand, chemical enhancement is based on charge transfer between metal and adsorbed molecules on plasmonic nanostructures. However, 
the chemical enhancement contribution is limited and its contribution is dependent on the chemical structure of the molecule $[17,18]$.

The plasmonic properties of metallic surfaces/nanoparticles include the resonance frequency of the SPs and electromagnetic field intensity at the surface. These properties strongly depend on their type, size, shape, composition, dimension (2D vs. 3D) and dielectric environment [19-22]. There is also great effort on the fabrication of nanocomposite thin film such as $\mathrm{Ag} / \mathrm{TiO}_{2}$ and $\mathrm{Ag} / \mathrm{ZrO}_{2}$ to obtain plasmonic nanomaterials [23-27] The plasmonic properties of the nanocomposite thin films depend on the size, shape, and density of Ag nanoparticles in the composite thin films. As aforementioned, the electromagnetic enhancement is the major contributor to the SERS enhancement mechanism. To tune the SPs, different size, shape, type, composition, and dimensions (2D vs. 3D) of plasmonic nanostructures have been extensively studied in SERS research. Upon the nanostructures, the plasmonic properties are tuned and the surface plasmons wavelengths and the electromagnetic field magnitude at the surface of nanostructures are altered. The experimental and theoretical studies confirmed that the SERS performance is maximized under the circumstances, in which the SP wavelength of the nanostructures $\left(\lambda_{\mathrm{SP}}\right)$ is between the wavelength of the excitation $\left(\lambda_{\text {exc }}\right)$ and the Raman signal wavelength $\lambda_{R S}$ [28-32]. Thus, the use of a proper excitation wavelength laser for SERS experiment is necessary to obtain an intense electromagnetic field on the nanostructures leading to higher SERS enhancement. Furthermore, it is commonly referred to as the SERS enhancement factor directly proportional to the fourth power of electromagnetic field intensity generated on the plasmonic nanostructures [33, 34]. Thus, the use of plasmonic nanostructures having intense electric field is also crucial for SERS experiments. There exist a few electromagnetic techniques to calculate the plasmonic properties of nanostructures such as discrete dipole approximation (DDA) [33, 35] and finite-difference time-domain (FDTD) [36], which are commonly used for theoretical calculations of the wavelength of SPs and intensity and the electromagnetic field distribution at the surface of plasmonic nanostructures.

Maximum SERS enhancement factors were obtained via controlling the plasmonic properties of the fabricated 3D nanostructures by several advanced techniques, for instance nanoholes [37-40], nanovoids [41-43], nanoclusters [44], and nanodomes $[45,46]$. The plasmonic properties of the nanoholes were tuned by the fabrication of nanostructures having different diameter and periodicity (hole spacing) to maximize the SERS enhancement $[37,40]$. With a similar manner, changing the diameter and depth in nanovoids tuned their plasmonic properties [41-43]. It was shown that the size, geometry, and interparticle spacing of the nanoclusters were changed to tune the nanoclusters' plasmonic properties [44], whereas interdome spacing of nanodomes were changed to obtain a higher SERS enhancement $[45,46]$.
Even if all these 3D well-defined nanostructures are capable of enhancing the SERS signals, they still lack simple, robust, large-scale, large-size, and cost-effective subtract manufacture procedures. The purpose of this study was to fabricate a simple, cost-effective, large-scale, and large-sized 3D plasmonic SERS substrate. In our study, we demonstrated a novel approach to fabricate tunable silver nanodomes (AgNDs). First, the convective assembly method was used for the latex particle uniform deposition on a glass slide. Nanovoids were constructed through polydimethylsiloxane (PDMS) by casting of the PDMS on the assembled latex particles. Finally, electrochemical method was used for the deposition of $\mathrm{Ag}$ to fill the nanovoids to obtain AgNDs. The structural property characterization of the fabricated AgNDs was performed using AFM and SEM. The optical property calculation of the AgNDs was made using the FDTD method with the aid of a commercial FDTD software (Lumerical FDTD Solutions) to investigate the plasmonic AgNDs properties. Optical nanostructure characterization was experimentally performed using SERS.

\section{Experimental Section}

\section{Fabrication of Plasmonic AgNDs}

To fabricate AgNDs, three distinct steps, connective assembly of latex particles, PDMS pouring, and silver deposition, were followed. In details, in Step 1, by using connective assembly method, spherical sulfate latex particles (Invitrogen, USA) having different diameters $(1600,1400,1200,1000,800$, 600 , and $400 \mathrm{~nm}$ ) were uniformly deposited on regular glass slides $[47,48]$. Experimental conditions of the method were optimized by changing concentration of the latex particles, suspension volume placed between two glass slides, and velocity of the stage used for the deposition of the particles. The proper conditions of the 1600-, 1400-, 1200-, and 1000-nm latex particles to assemble uniformly were acquired upon when the concentration of the particles, volume of the suspension, and the stage velocity were set as $0.8 \%, 40 \mu \mathrm{L}$, and $1 \mu \mathrm{m} / \mathrm{s}$, respectively. For the smaller-sized latex particles such as 800,600 , and $400 \mathrm{~nm}$, however, the optimum particle concentration, suspension volume, and stage velocity conditions were identified as $1.6 \%, 40 \mu \mathrm{L}$, and $0.8 \mu \mathrm{m} / \mathrm{s}$, respectively. Step 2: PDMS (Dow Corning, USA) nanovoids were obtained by pouring PDMS on top of the assembled latex particles and curing polymer mixture for $45 \mathrm{~min}$ at $70{ }^{\circ} \mathrm{C}$. The PDMS was peeled off and washed with dichloromethane (Sigma-Aldrich, USA) to remove residues of the latex particles to prepare nanovoids on the PDMS. For the final fabrication process, Step 3, AgNDs with different heights and diameters were obtained by filling PDMS nanovoids with the deposition of Ag. 
The surface of PDMS was deposited with a very thin $\mathrm{Cr}$ layer $(\sim 10 \mathrm{~nm})$ by vapor deposition technique to make it conductive and enhancing adherence of Ag to the PDMS surface. Then, the modified PDMS nanovoids were filled with deposition of Ag. The deposition of Ag was performed galvanostatically by applying a constant $3.5-\mathrm{mA}$ current until ensuring all of the nanovoids were filled with $\mathrm{Ag}(\sim 4 \mu \mathrm{m})$ for all nanovoids. The thicknesses of the coatings were theoretically calculated through the deposition charge taking into account the current efficiency of $100 \%$ ( NiCu HE). Since Ag is a soft and breakable metal, the top of the Ag layer was thickened by the electrodeposition of a thicker nickel (Ni) top-film which is a harder metal. The Ni electrodeposition was performed galvanostatically by applying $2.5 \mathrm{~mA}$ for $1800 \mathrm{~s}$ and followed $10 \mathrm{~mA}$ for $5400 \mathrm{~s}$. To avoid excess hydrogen gas evolution at the cathode and removing the metal from PDMS, a lower current (2.5 mA) was initially applied for the Ni electrodeposition. Soluble Ag and Ni samples were used as anodes during the electrochemical deposition of $\mathrm{Ag}$ and $\mathrm{Ni}$, respectively. Chemical compositions of $\mathrm{Ag}$ and $\mathrm{Ni}$ baths are given below.

Ag bath solution: $0.1 \mathrm{M} \mathrm{AgNO}_{3}+0.1 \mathrm{M} \mathrm{KNO}_{3}+1 \mathrm{M}$ $\mathrm{NH}_{3}$.

$\mathrm{Ni}$ bath solution: $30 \% \mathrm{NiSO}_{4} \cdot 7 \mathrm{H}_{2} \mathrm{O}+1 \% \mathrm{NiCl} \cdot 6 \mathrm{H}_{2} \mathrm{O}+$ $1.25 \% \mathrm{H}_{3} \mathrm{BO}_{3}$.

After the Ag/Ni deposition, the processed PDMS surface was removed from the deposition bath; the Ni-supported AgNDs were peeled off from the PDMS surface with an acidic solution treatment to dissolve $\mathrm{Cr}$, carefully washed with distilled water to remove the impurities and residual chemicals, degreased with absolute ethanol, and at the end dried with nitrogen gas. EDX measurements were obtained to assess the purity of AgNDs fabricated using this approach. The results showed that the AgNDs were as high as 99.9\% pure (results not shown here).

\section{Structural Characterizations}

The structural characterization of the assembled latex particles, PDMS nanovoids, and AgNDs were performed via SEM and AFM. A JEOL 6510 and Park Systems XE-100E instruments were used to obtain the SEM and AFM images.

\section{AgNDs Plasmonic Properties Simulations}

The optical property calculation of the AgNDs is made by using finite difference time domain method with the aid of a commercial FDTD software package (Lumerical FDTD Solutions). The FDTD method is a very well-known numerical analysis technique used for modeling computational electrodynamics. This technique is one of the grid-based differential numerical modeling methods. The FDTD method is a direct space-time approach. In this method, an initial field is sent to the domain of calculations and its temporal and spatial evolution is analyzed. The time-dependent Maxwell's equations in differential form are discretized to the space and time partial derivatives to obtain finite-difference equations. Finally, these equations are solved. For the calculation of reflection and electric field enhancement values, periodic boundary conditions are used in the x-y plane to simulate a periodic array. While, perfectly matched layer (PML) is used for the boundaries in the z-direction. The upper boundary is set to air (background index = 1) and lower boundary is set to Ag whose optical properties are taken from Palik's handbook. To excite the system, a unit plane wave propagating in the $-\mathrm{Z}$ direction with a normal incident is used. For the calculation of absorption, extinction, and scattering values, boundary conditions in the $x-y$ plane and the source used are changed. Periodic antisymmetric and symmetric boundary conditions are chosen at the $\mathrm{x}$ and $\mathrm{y}$ planes, respectively. Total-field scattered-field (TFSF) source whose $x-y$ dimensions are equal to dimensions of the unit cell of simulated structure is used for the excitation. The injection of the source is performed in the $\mathrm{z}$ direction with a normal incident. All simulations are performed in the wavelength range of 0.3 to $1.3 \mu \mathrm{m}$.

\section{SERS Measurements}

4-aminothiphonel (4-ATP) (Sigma-Aldrich, USA) was used for the evaluation of the SERS performance of the fabricated AgNDs. For this purpose, the fabricated AgNDs were treated with ATP (1 mM) dissolved in ethanol for an hour. Afterwards, the AgNDs surfaces were washed with ethanol and water. The SERS measurements were performed with a Renishaw InVia Reflex Raman Microscopy System (Renishaw Plc., U.K.) equipped with 785-nm lasers. A laser at $785 \mathrm{~nm}$ and a $\times 50$ objective (numerical aperture: 0.75 ) with a laser power of $1.5 \mathrm{~mW}$ was used for all experiments. The exposure time and accumulation were $1 \mathrm{~s}$ and 1 , respectively. To calculate the SERS enhancement factor of the AgNDs, the Raman spectra of a $5 \mu \mathrm{L}$ of rhodamine $6 \mathrm{G}(0.1 \mathrm{M})$ on a $\mathrm{CaF}_{2}$ slide and SERS spectra from the surface of AgNDs treated with a $5 \mu \mathrm{L}$ of rhodamine $6 \mathrm{G}\left(1.0 \times 10^{-6} \mathrm{M}\right)$ were acquired. All presented SERS spectra of 4-ATP and rhodamine $6 \mathrm{G}$ were the average of ten spectra acquired from randomly selected spots on the sample. The percent coefficient of variation (CV\%) of the SERS spectra was in the range of $8-13 \%$ for the fabricated AgNDs which is acceptable for SERS experiments.

\section{Results and Discussion}

In this study, we designed a novel 3D plasmonic AgNDs involving three simple main steps which minimize the cost and allow large-scale and large-size substrate production. First, convective assembly technique was used to uniformly 
assemble the latex particles spanning size between 1600 and $400 \mathrm{~nm}$ on a glass slide to obtain a template to prepare nanovoids on the PDMS surfaces. After the latex particles were assembled on a slide, PDMS was poured and cured on the assembled latex particles. The cured PDMS was then peeled off to prepare nanovoids on the surface of PDMS with different depths and diameters depending on the diameter of the used latex particles to obtain nanovoids. Finally, the nanovoids having different depths and diameters were filled with $\mathrm{Ag}$ and $\mathrm{Ni}$ with electrochemical deposition to obtain plasmonic AgNDs having a variety of diameters and heights depending on the used PDMS template, respectively. Figure 1a illustrates each step of the AgND fabrication with the support of the post-SEM images of the individual three fabrication steps of plasmonic AgNDs (Fig. 1b).

SEM images were taken for the characterization of the assembled latex particles, PDMS nanovoids, and fabricated AgNDs. Figure 2 shows the SEM images of assembled latex particles $(1200 \mathrm{~nm})$, PDMS nanovoids, and AgNDs fabricated by the electrochemical deposition of $\mathrm{Ag}$ and $\mathrm{Ni}$ on the PDMS surface.

The diameter of the latex particles was changed to tune the structural properties of the AgNDs. Larger diameter and deeper nanovoids on the PDMS surface were obtained as larger diameter of latex particles was used. However, smaller diameter and shallower nanovoids were obtained on the PDMS surfaces as smaller particles were used. To obtain AgNDs having different plasmonic properties, PDMS nanovoids were filled with deposition of $\mathrm{Ag}$. When the larger-diameter (deeper, as well) PDMS surfaces were used for the fabrication of AgNDs as a template, AgNDs having a larger diameter (higher height) were fabricated. While by using the smallerdiameter (shallower) PDMS surfaces for the fabrication of AgNDs as a template, AgNDs having smaller diameter (lower height) were fabricated. In this manner, AgND plasmonic properties were able to be tuned by changing the heights and the diameters of the AgNDs. The SEM images obtained from all the assembled latex particles, the PDMS nanovoids, and the fabricated AgNDs used in this study are shown in Fig. 3.

As seen in Fig. 3, the deposited latex particles with different diameters is closely assembled and uniform on the regular glass slides. PDMS nanovoids having different depths and diameters can be fabricated using different diameters of latex particles. Further, AgNDs having different heights and diameters can be fabricated by the electrochemical deposition of Ag.
Fig. 1 Schematic illustration of the steps to fabricate tunable plasmonic AgNDs (a) and SEM images of assembled latex particles, PDMS nanovoids, and AgNDs (b)

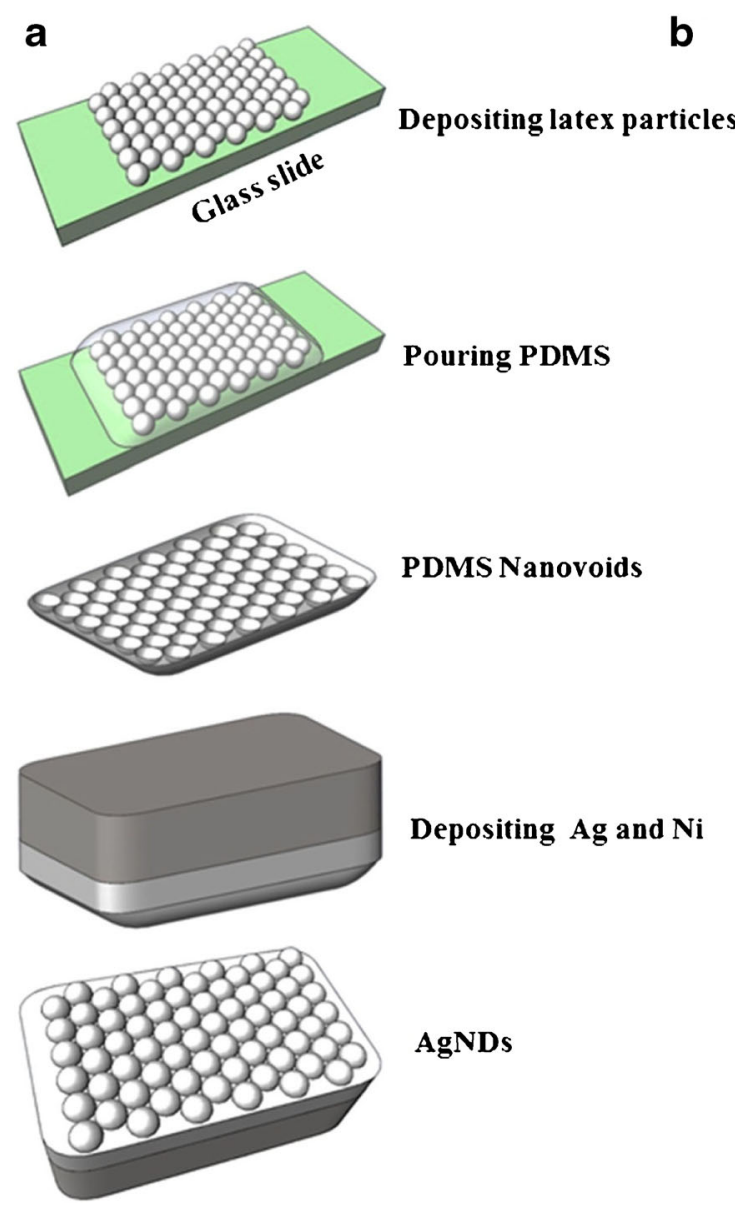

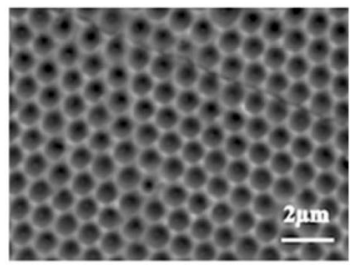

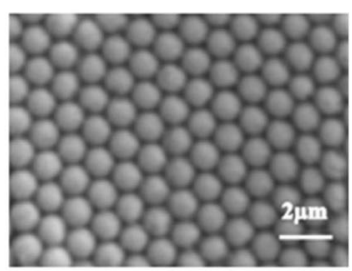


Fig. 2 SEM images obtained from the assembled latex particles (1200 nm), PDMS nanovoids, and fabricated AgNDs

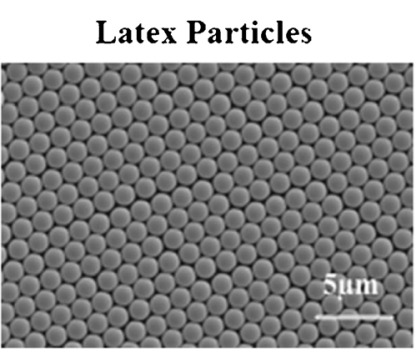

AgNDs

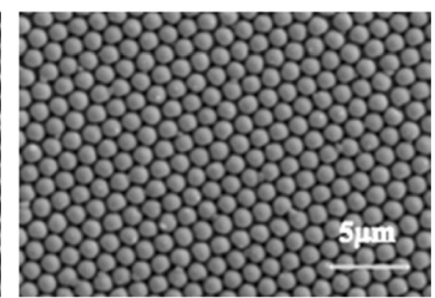

Lower magnification of SEM images of AgNDs fabricated using 600- and 1200-nm latex particles (Fig. 4) shows the uniformity of the structures with a larger scale bar. As seen in the figure, by following this simple fabrication method provided a cost-effective, large-scale, and large-sized substrate production.
AFM images were obtained from the assembled latex particles, PDMS nanovoids, and fabricated AgNDs to demonstrate how the topography changes depending on the diameter of latex particles (see Fig. 5).

As we discussed earlier, when the smaller latex particles were used, smaller diameter and shallower nanovoids were
Fig. 3 SEM images obtained from all the assembled latex particles, PDMS nanovoids, and fabricated AgNDs used in this study

\section{Latex Particles PDMS Nanovoids AgNDs}

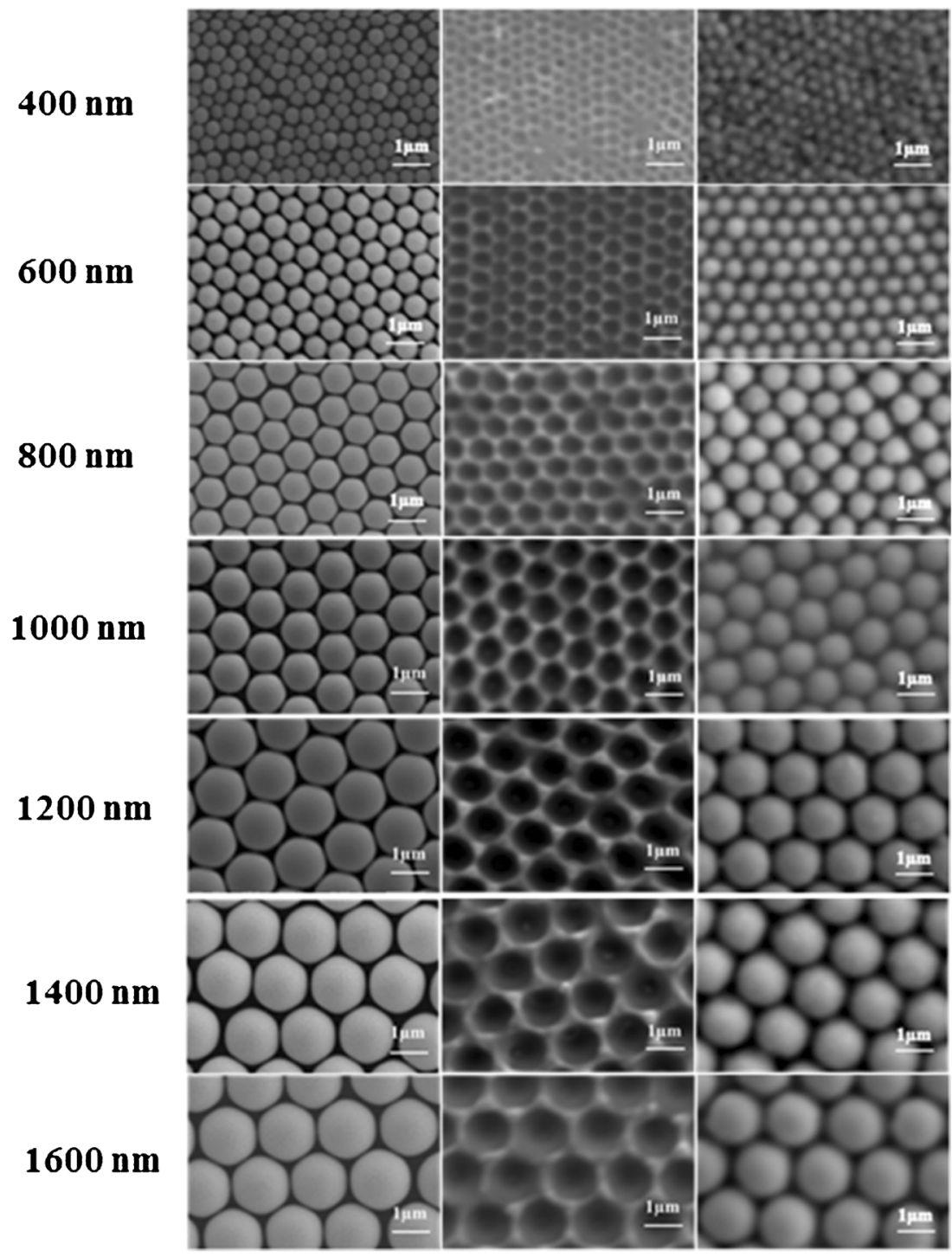


Fig. 4 SEM images obtained from the fabricated AgNDs using 600- and 1200-nm latex particles
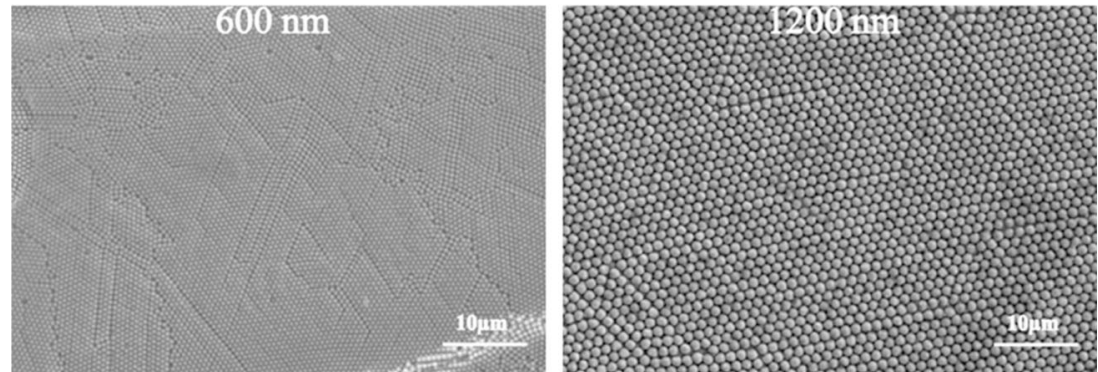

fabricated. Thus, when the lower-depth PDMS nanovoids were used, smaller diameter with low height AgNDs was obtained. On the other hand, when the larger latex particles were used, larger diameter and deeper nanovoids were obtained. These nanovoids were used as a mold to fabricate larger diameter and higher height owning AgNDs The size effect of latex particles on the fabrication of AgNDs after individual steps can be seen clearly in Fig. 5. Since SEM images do not allow to get detailed topographic information such as the size of depth and height, AFM was used to characterize the surface topography of the AgND fabrications to acquire the height and diameter of the structures as shown in Fig. 6.

The AgND diameter and homogeneity obtained with AFM images are consistent with the SEM images (Fig. 6a). The AgNDs can clearly be seen in the 3D AFM image (Fig. 6b). The AFM line analysis was presented to measure the diameter and height of AgNDs and evaluate the uniformity of the structure. The AgND diameter and height were measured as around 1000 and $300 \mathrm{~nm}$, respectively, when the diameter of latex particles was $1000 \mathrm{~nm}$. Moreover, it seems to have a higher uniformity across to AgNDs surface (Fig. 6c). The structural characterization results obtained with the measurement of 10 AgNDs through the AFM images can be seen in Table 1.

Optical properties of the fabricated AgNDs was theoretically calculated using the FDTD method. To explore the plasmonic properties (the surface plasmon wavelength and the electromagnetic field intensity) of AgNDs depending on the AgND diameters and heights, FDTD simulation was performed. We calculated LSPR $\left(\lambda_{\mathrm{LSPR}}\right)$, including the scattering $\left(\mathrm{C}_{\mathrm{sca}}\right)$, absorption $\left(\mathrm{C}_{\mathrm{abs}}\right)$, and extinction cross-sections $\left(\mathrm{C}_{\text {ext }}\right)$, of the fabricated AgNDs as a wavelength function depending on the AgND diameters and heights. The calculated scattering, absorption, and extinction cross-sections of the AgNDs fabricated using 1000-nm latex particles is shown in Fig. 7a. Figure $7 \mathrm{~b}$ shows the scattering cross-sections of the AgNDs having different diameters and heights.

We demonstrated the LSPR spectra for AgNDs fabricated using 1000-nm latex particles. The maximum broad resonance peaks for scattering and extinction at around $800 \mathrm{~nm}$ were obtained from the AgND cross-sections. This resonance

\section{Latex Particles}

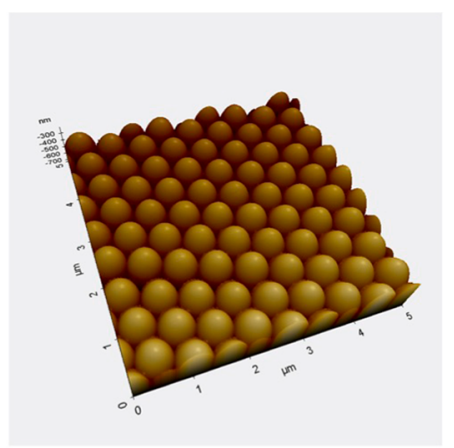

$1600 \mathrm{~nm}$

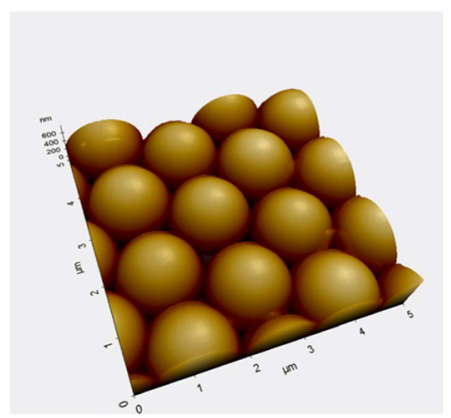

PDMS Nanovoids
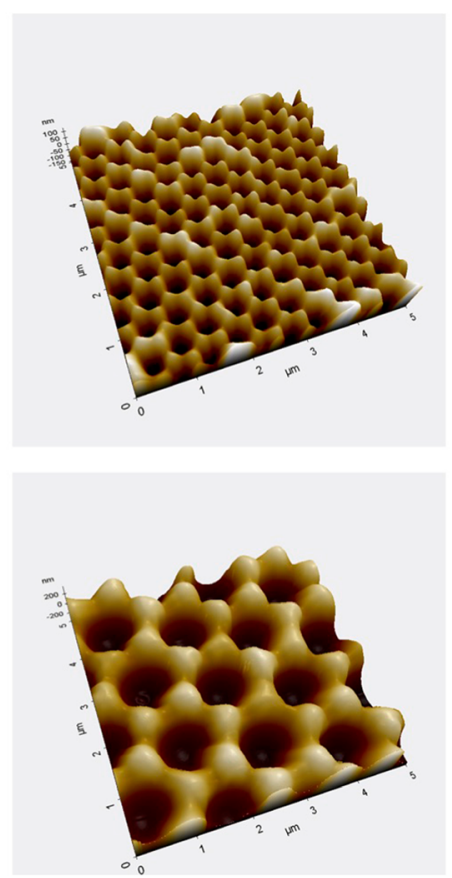

AgNDs
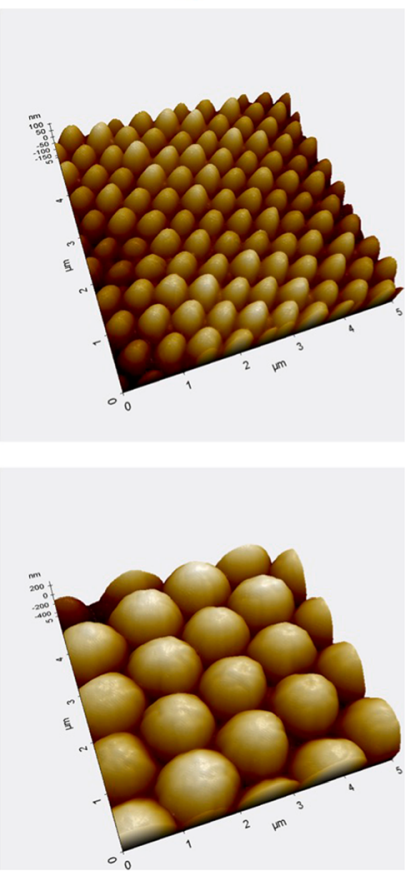

Fig. 5 3D AFM images of latex thin films, PDMS nanovoids, and AgNDs 


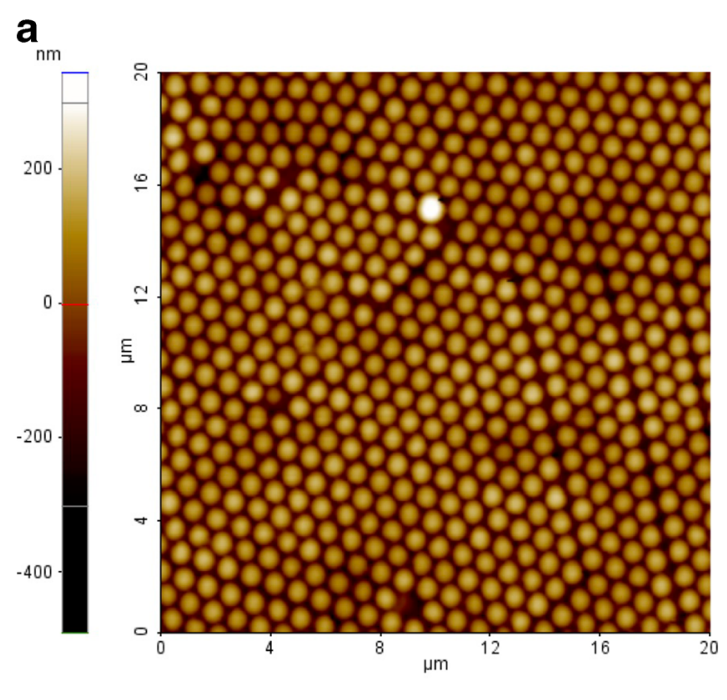

b
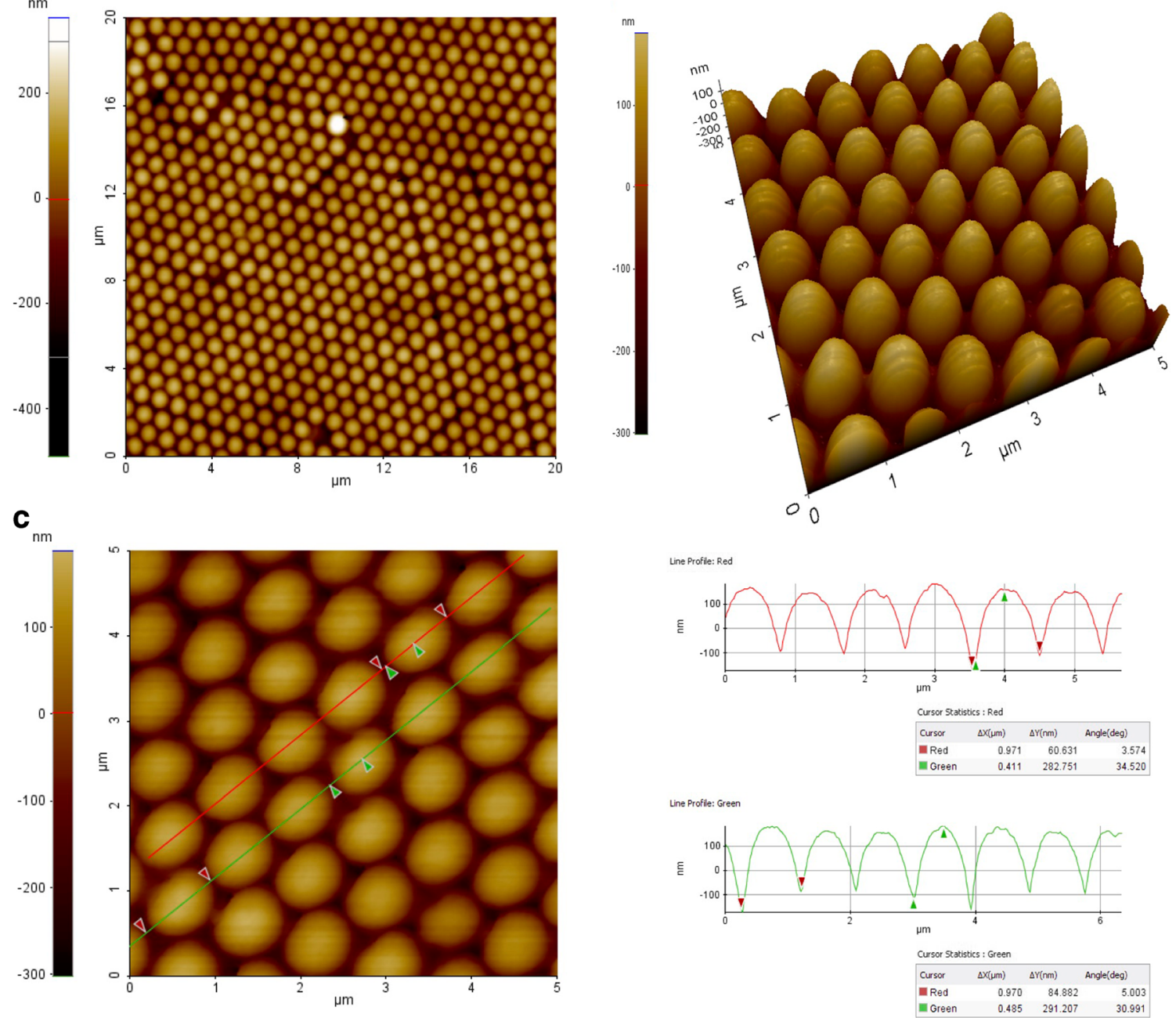

Fig. 6 2D (a), 3D AFM images (b), and line analysis across the red and green lines (c) of AgNDs fabricated using 1000-nm latex particles

wavelength is very close to the excitation wavelength. On the other hand, there was no maximum resonance peak for absorption as seen in Fig. 7a. Similar results were obtained for the FDTD calculated absorption, scattering, and extinction spectra for other fabricated AgNDs. The AgND scattering spectra position is strongly dependent on the latex particle diameter used for the AgND fabrication. When the AgND diameter and height was increased, the AgND scattering wavelength shifts to longer wavelength (see Fig. 7b). As a result, when the diameter of latex particles used for the AgND fabrication are changed, LSPR wavelengths are changed allowing to tune plasmonic properties. Furthermore, the intensity of electromagnetic field enhanced around and on the plasmonic nanostructures. To estimate the E- field distribution between adjacent AgNDs, FDTD was also used for the E-field calculation. E-field distributions are plotted for AgNDs with experimental excitation wavelength at $785 \mathrm{~nm}$ which is a laser line on the Raman spectrometer. Figure 8 shows the E-field distribution (in log scale) between adjacent AgNDs depending on the diameters and heights.

When the latex particles of smaller size were used (Fig. 8 top), the AgNDs exhibited poor E-field intensity between adjacent AgNDs due to shorter LSPR wavelengths compared to the excitation wavelength $(785 \mathrm{~nm}$ ) (see Fig. 7b). However, when the latex particle diameter was increased up to $1000 \mathrm{~nm}$, an increment in the E-field intensity was observed (data not shown). The maximum E-field was obtained between adjacent AgNDs when

Table 1 Results of structural characterization obtained from AFM images

\begin{tabular}{llllllll}
\hline Latex particle diameter & $400 \mathrm{~nm}$ & $600 \mathrm{~nm}$ & $800 \mathrm{~nm}$ & $1000 \mathrm{~nm}$ & $1200 \mathrm{~nm}$ & $1400 \mathrm{~nm}$ & $1600 \mathrm{~nm}$ \\
\hline AgND diameter & $370 \pm 21 \mathrm{~nm}$ & $519 \pm 24 \mathrm{~nm}$ & $730 \pm 46 \mathrm{~nm}$ & $965 \pm 15 \mathrm{~nm}$ & $1150 \pm 24 \mathrm{~nm}$ & $1362 \pm 24 \mathrm{~nm}$ & $1405 \pm 31 \mathrm{~nm}$ \\
AgND height & $69 \pm 13 \mathrm{~nm}$ & $102 \pm 22 \mathrm{~nm}$ & $140 \pm 18 \mathrm{~nm}$ & $280 \pm 12 \mathrm{~nm}$ & $348 \pm 25 \mathrm{~nm}$ & $434 \pm 36 \mathrm{~nm}$ & $452 \pm 40 \mathrm{~nm}$ \\
\hline
\end{tabular}



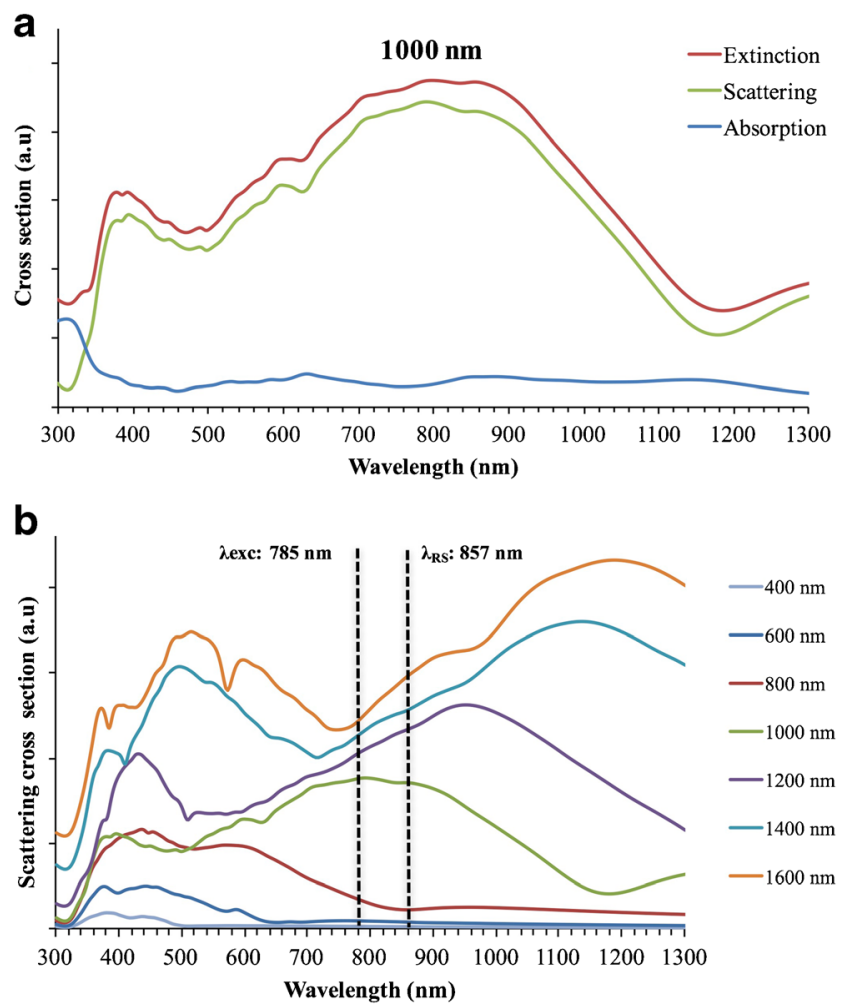

Fig. 7 FDTD calculated scattering, absorption, and extinction crosssections of the AgNDs fabricated using 1000-nm latex particles (a) and scattering cross-sections of all fabricated AgNDs (b) the 1000-nm latex particles were used for the AgND fabrication (Fig. 8 middle). This is due to that the wavelength of the calculated scattering spectrum is close to the excitation wavelength that provides the highest E-field. Moreover, as the size of latex particles was increased, a decrease in the intensity of E- field was obtained (Fig. 8 bottom), because the wavelength of calculated scattering spectrum is far from the excitation wavelength which provides poor E-field (Fig. 7b).

Finally, SERS was used for the experimental optical property characterization of the fabricated AgNDs. The SERS spectra were obtained from 4-ATP molecules and used to assess the SERS performance of the fabricated AgNDs. Figure 9 shows the SERS spectra of 4-ATP molecules assembled on the AgNDs (A) and changes in peak intensity at $1080 \mathrm{~cm}^{-1}$ for AgNDs having different diameters and heights (B). The different SERS spectra intensities were acquired for AgNDs having different diameters and heights due to the different plasmonic properties. In this study, the intense peak at $1080 \mathrm{~cm}^{-1}$ was used to calculate the $\lambda_{\mathrm{RS}}$ and found to be $857 \mathrm{~nm}$ as the $\lambda_{\text {exc }}$ is $785 \mathrm{~nm}$. It is well known that the SERS enhancement is maximized when the $\lambda_{\mathrm{LSPR}}$ of plasmonic nanostructures is between $\lambda_{\mathrm{RS}}$ and $\lambda_{\text {exc }}$. The results confirmed that the maximum SERS intensity was achieved when 1000-nm latex particles were used for the fabrication of AgNDs due to the $\lambda_{\text {LSPR }}$ being between $\lambda_{\mathrm{RS}}$ and $\lambda_{\text {exc }}$. In the literature, it is commonly referred to as the SERS enhancement factor which is directly proportional to the fourth power of electromagnetic field intensity generated on the plasmonic nanostructures [7, 45] The

\section{Exc: 785nm}
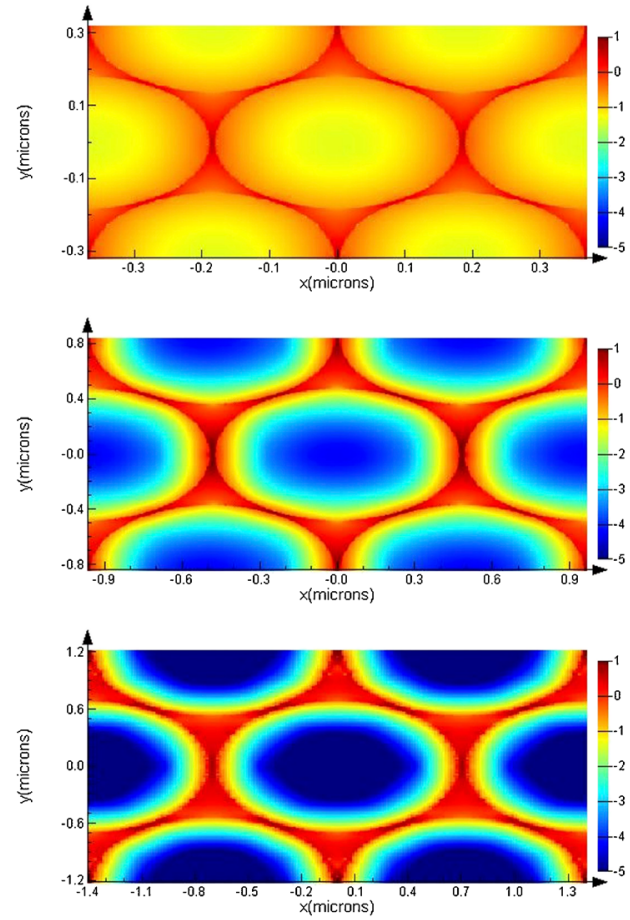

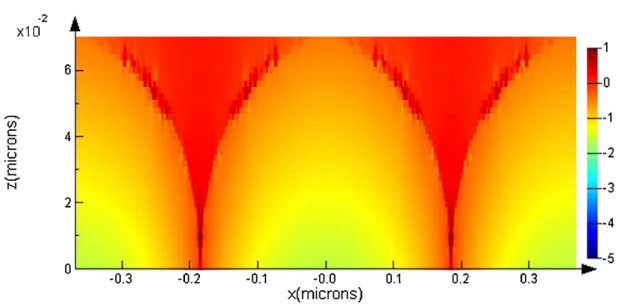

$400 \mathrm{~nm}$

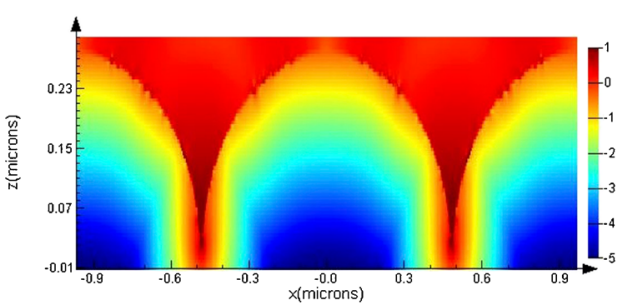

$1000 \mathrm{~nm}$

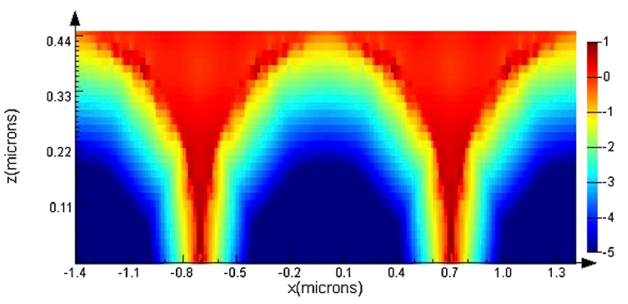

$1600 \mathrm{~nm}$

Fig. 8 FDTD calculated E-field distribution-adjacent AgNDs depending on the diameters and heights 
Fig. 9 SERS spectra of 4-ATP molecules assembled on AgNDs with different heights and diameters depending on the latex particles used for the AgND fabrication (a), and changes in the SERS intensity of the peak at $1080 \mathrm{~cm}^{-1}$ depending on the AgNDs heights and diameters (b) a
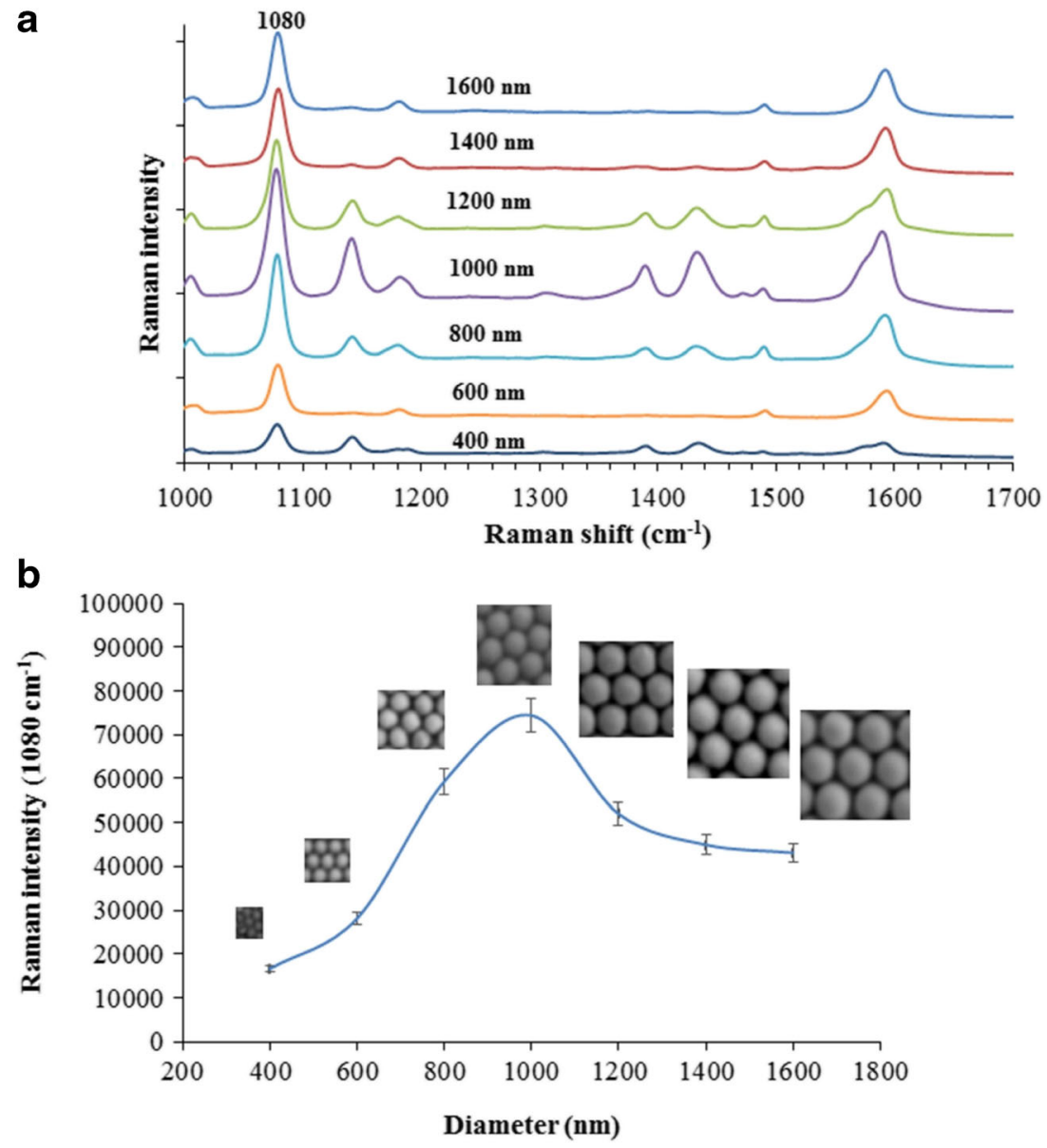

maximum SERS activity was also obtained due to having maximum E-field between adjacent AgNDs when the1000 nm latex particles were used for the fabrication (Fig. 8 middle).

Lastly, the SERS enhancement factor was calculated using rhodamine $6 \mathrm{G}$ for the AgNDs having the highest SERS activity. The peak at $1512 \mathrm{~cm}^{-1}$ was chosen to estimate the analytical enhancement factor (AEF) of the AgNDs. Solution (0.1 M and $1.0 \times 10^{-6} \mathrm{M}$ ) of rhodamine $6 \mathrm{G}$ was used to obtain bulk

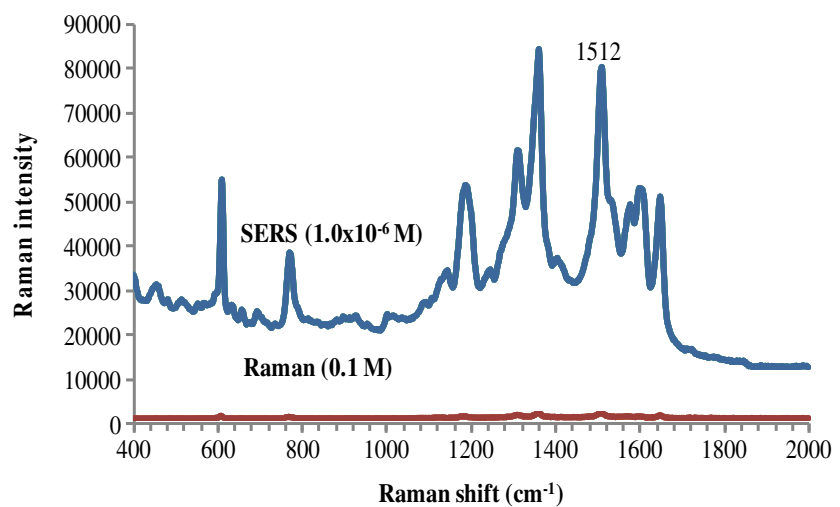

Fig. 10 Bulk Raman and SERS spectra obtained from the solution of rhodamine $6 \mathrm{G}\left(0.1 \mathrm{M}\right.$ and $\left.1.0 \times 10^{-6} \mathrm{M}\right)$ dropped on $\mathrm{a} \mathrm{CaF}_{2}$ slide and AgNDs
Raman and SERS spectra, respectively. The AEF was calculated by using the formula $\mathrm{I}_{\mathrm{SERS}} / \mathrm{I}_{\mathrm{RS}} \mathrm{x} \mathrm{C}_{\mathrm{RS}} / \mathrm{C}_{\mathrm{SERS}}$ [49]. Figure 10 shows the comparison of Raman spectrum of rhodamine $6 \mathrm{G}$ and SERS spectrum of rhodamine $6 \mathrm{G}$ dropped on AgNDs fabricated using 1000-nm latex particles.

The SERS spectra intensity ratio and the concentration factor were 60.5 and $1.0 \times 10^{5}$, respectively. The maximum AEF was found to be $6.05 \times 10^{6}$ from this formulation. In addition,

Fig. 11 Comparison of SERS spectra of 4-ATP molecules assembled on AgNDs and Ag thin film

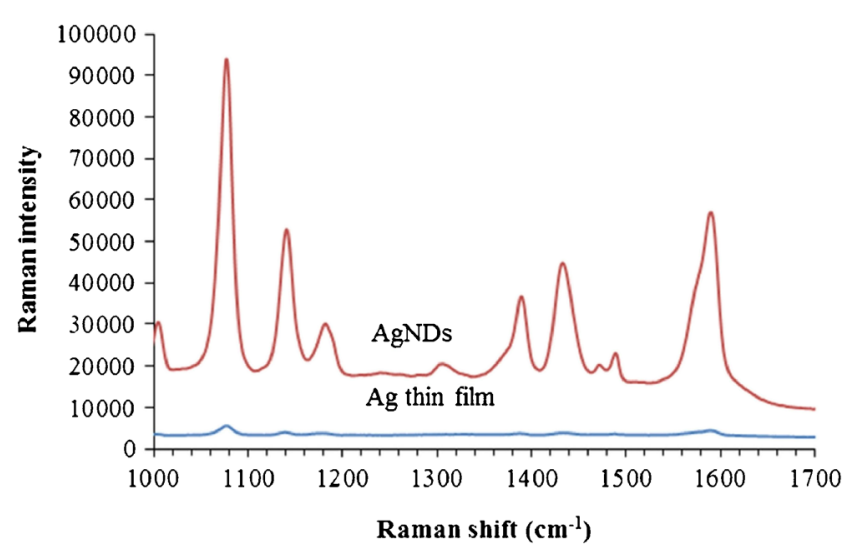


the AgNDs having the highest SERS performance was compared to the Ag thin film surface. Approximately two orders of magnitude improvement in the SERS activity on the AgNDs were observed (Fig. 11) due to the intense E-field generated between adjacent AgNDs.

\section{Conclusions}

In conclusion, we reported a novel method to fabricate AgNDs having tunable plasmonic properties by changing of heights and diameters of AgNDs. The latex particles with different diameters were used to prepare PDMS nanovoids with different depths and diameters that were used as a template for the fabrication of AgNDs having different heights and diameters with the electrochemical deposition of Ag. The experimental and theoretical results demonstrated that the SERS activity of AgNDs was strongly dependent on the heights and diameters of the structures. The maximum SERS enhancement was obtained (AEF: $6.05 \times 10^{6}$ ) with the latex particles having $1000 \mathrm{~nm}$ diameter used for the fabrication of AgNDs due to the highest E-field distributions between the adjacent AgNDs and the presence of AgNDs $\lambda_{\text {LSPR }}$ between $\lambda_{\text {exc }}$ and $\lambda_{\text {RS }}$. The fabricated AgNDs having the highest SERS enhancement can be used for SERS-based detection and identification of chemical and biological structures.

Acknowledgments The authors acknowledge the financial support of the Scientific and Technological Research Council of Turkey (TUBITAK) (Project No:114Z414), Gaziantep University, and Bingöl University. H.C. also acknowledges partial support from the Turkish Academy of Sciences.

\section{References}

1. Stewart ME, Anderton CR, Thompson LB, Maria J, Gray SK, Rogers JA, Nuzzo RG (2008) Nanostructured plasmonic sensors. Chem Rev 108:494-521

2. Atwater HA (2007) The promise of plasmonics. Sci Am 296:56-63

3. Ozbay E (2006) Plasmonics: merging photonics and electronics at nanoscale dimensions. Science 311:189-193

4. Maier SA, Atwater HA (2005) Plasmonics: localization and guiding of electromagnetic energy in metal/dielectric structures. J Appl Phys 98:011101

5. Van Duyne RP (2004) Molecular plasmonics. Science 306:985986

6. Anker JN, Hall WP, Lyandres O, Shah NC, Zhao J, Van Duyne RP (2008) Biosensing with plasmonic nanosensors. Nat Mater 7:442453

7. Haes AJ, Van Duyne RP (2004) A unified view of propagating and localized surface plasmon resonance biosensors. Anal Bioanal Chem 379:920-930

8. Brockman JM, Nelson BP, Corn RM (2000) Surface plasmon resonance imaging measurements of ultrathin organic films. Ann Rev Phys Chem 51:41-63

9. Schatz GC, Van Duyne RP (2002) Handbook of vibrational spectroscopy. Wiley, New York
10. Fleischman M, Hendra PJ, McQuillan AJ (1974) Raman-spectra of pyridine adsorbed at a silver electrode. Chem Phys Lett 26:163-166

11. Jeanmaire DL, Van Duyne RP (1977) Surface raman spectroelectrochemistry 1 . Heterocyclic, aromatic, and aliphaticamines adsorbed on anodized silver electrode. J Electroanal Chem 84:1-20

12. Albrecht MG, Creighton JA (1977) Anomalously intense Raman spectra of pyridine at a silver electrode. J Am Chem Soc 99:52155217

13. Moskovits M (1978) Surface roughness and the enhanced intensity of Raman scattering by molecules adsorbed on metals. J Chem Phys 69:1459-1461

14. Moskovits M (1985) Surface-enhanced spectroscopy. Rev Mod Phys 57:783-826

15. Otto A (1984) In: Cardona M, Guntherodt G (eds) In light scattering in solids IV. Electronic scattering, spin effects, SERS and morphic effects. Springer-Verlag, Berlin

16. Persson BNJ (1981) On the theory of surface-enhanced Raman scattering. Chem Phys Lett 82:561-565

17. Haynes C, Mcfarland AD, Van Duyne RP (2005) Surface-enhanced Raman spectroscopy. Anal Chem 77:339A-346A

18. Campion A, Kambhampati P (1998) Surface-enhanced Raman scattering. Chem Soc Rev 27:241-250

19. Kelly KL, Coronado E, Zhao LL, Schatz GC (2003) The optical properties of metal nanoparticles: the influence of size, shape, and dielectric environment. J Phys Chem B 107:668-677

20. Hutter E, Fendler JH (2004) Exploitation of localized surface plasmon resonance. Adv Mater 16:1685-1706

21. Henzie J, Lee J, Lee MH, Hasan W, Odom TW (2009) Nanofabrication of plasmonic structures. Annu Rev Phys Chem 60:147-165

22. Lu X, Rycenga M, Skrabalak SE, Wiley B, Xia Y (2009) Chemical synthesis of novel plasmonic nanoparticles. Annu Rev Phys Chem 60:167-192

23. Kumar M, Reddy GB (2010) Tailoring surface plasmon resonance in ag: $\mathrm{ZrO}_{2}$ nanocomposite thin films. Phys E 43:470-474

24. Kumar M, Suchand Sandeep CS, Kumar G, Mishra YK, Philip R, Reddy GB (2014) Plasmonic and nonlinear optical absorption properties of ag:ZrO2 nanocomposite thin films. Plasmonics 9:129-136

25. Kumar M, Kumar T, Kumar Avasthi D (2015) Study of thermal annealing induced plasmonic bleaching in $\mathrm{Ag}$ : $\mathrm{TiO}_{2}$ nanocomposite thin films. Scr Mater 105:46-49

26. Kumar M, Reddy GB (2016) Stability-inspired entrapment of Ag nanoparticles in $\mathrm{ZrO}_{2}$ thin films. Plasmonics 11:261-267

27. Kumar M, Jangid T, Panchal V, Kumar P, Pathak A (2016) Effect of grazing angle cross-ion irradiation on Ag thin films. Nanoscale Res Lett 11:454

28. Haynes CL, Yonzon CR, Zhang X, Van Duyne RP (2005) Surfaceenhanced Raman sensors: early history and the development of sensors for quantitative Biowarfare agent and glucose detection. J Raman Spectrosc 36:471-484

29. Grand J, de la Chapelle ML, Bijeon L-J, Adam P-M, Vial A, Royer $P$ (2005) Role of localized surface plasmons in surface-enhanced Raman scattering of shape-controlled metallic particles in regular arrays. Phys Rev B 72:033407

30. Haynes CL, Van Duyne RP (2003) Plasmon-sampled surface-enhanced Raman excitation spectroscopy. J Phys Chem B 107:74267433

31. Felidj N, Aubard J, Lévi G, Krenn JR, Salerno G, Schider G, Lamprecht B, Leitner A, Aussengg FR (2002) Controlling the optical response of regular arrays of gold particles for surfaceenhanced Raman scattering. Phys Rev B 65:075419

32. McFarland AD, Young MA, Dieringer JA, Van Duyne RP (2005) Wavelength-scanned surface-enhanced Raman excitation spectroscopy. J Phys Chem B 109:11279-12285 
33. Haes AJ, Haynes CL, McFarland AD, Schatz GC, Van Duyne RP, Zou S (2005) Plasmonic materials for surface-enhanced sensing and spectroscopy. MRS Bull 30:368-375

34. Stiles PL, Dieringer JA, Shah NC, Van Duyne RP (2008) Surfaceenhanced Raman spectroscopy. Annu Rev Anal Chem 1:601-626

35. Yang WH, Schatz GC, Van Duyne RP (1995) Discrete dipole approximation for calculating extinction and Raman intensities for small particles with arbitrary shapes. J Chem Phys 103:869-875

36. Gray SK, Kupta T (2003) Propagation of light in metallic nanowire arrays: finite-difference time-domain studies of silver cylinders. Phys Rev B 68:045415

37. Lee SH, Bantz KC, Lindquist NC, Oh SH, Haynes CL (2009) Selfassembled plasmonic nanohole arrays. Langmuir 25:13685-13693

38. Yu Q, Guan P, Qin D, Golden G, Wallace PM (2008) Inverted sizedependence of surface-enhanced Raman scattering on gold nanohole and nanodisk arrays. Nano Lett 8:1923-1928

39. Brolo AG, Arctander E, Gordon R, Leathem B, Kavanagh KL (2004) Nanohole-enhanced Raman scattering. Nano Lett 4:20152018

40. Yu Q, Braswell S, Christin B, Xu J, Wallace PM, Gong H, Kaminsky D (2010) Surface-enhanced Raman on gold quasi-3D nanostructure and 2D nanohole arrays. Nanotechnology 21:35530

41. Cintra S, Abdelsalam ME, Bartlett PN, Baumberg JJ, Kelf TA (2006) Sculpted substrates for SERS. Faraday Discuss 132:191-199
42. Baumberg JJ, Kelf TA, Sugawara Y, Cintra S, Abdelsalam ME, Bartlett PN, Russell AE (2005) Angle-resolved surface-enhanced Raman scattering on metallic nanostructured plasmonic crystals. Nano Lett 5:2262-2267

43. Lang X, Qiu T, Yin Y, Kong F, Si L, Hao Q, Chu PK (2012) Silver nanovoid arrays for surface-enhanced Raman scattering. Langmuir 28:8799-8803

44. Ye J, Wen F, Sobhani H, Lassiter JB, Dorpe PV, Nordlander P, Halas NJ (2012) Plasmonic nanoclusters: near field properties of the Fano resonance interrogated with SERS. Nano Lett 12:1660 1667

45. Wu H-Y, Choi CJ, Cunningham BT (2012) Plasmonic nanogapenhanced Raman scattering using a resonant nanodome array. Small 8:2878-2885

46. Choi CJ, Xu Z, Wu H-Y, Liu GL, Cunningham BT (2010) Surfaceenhanced Raman nanodomes. Nanotechnology 21:415301

47. Denkov ND, Velev OD, Kralchevsky PA, Ivanov IB, Yoshimura H, Nagayama K (1992) Mechanism of formation of two-dimensional crystals from latex particles on substrates. Langmuir 8:3183-3190

48. Denkov ND, Velev OD, Kralchevsky PA, Ivanov IB, Yoshimura H, Nagayama K (1993) Two-dimensional crystallisation. Nature 361: 26

49. Le Ru EC, Blackie E, Meyer M, Etchegoin PG (2007) Surface enhanced Raman scattering enhancement factors: a comprehensive study. J Phys Chem C 111:13794-13803 